\title{
RESPIRATORY CARE.
}

Systematic Review

Effect of Prone Positioning on Clinical Outcomes of Non-Intubated Subjects with COVID-19: A Comparative Systematic Review and Meta-Analysis

https://doi.org/10.4187/respcare.09362

Cite as: RESPCARE 2021; 10.4187/respcare.09362

Received: 3 June 2021

Accepted: 8 November 2021

This Fast Track article has been peer-reviewed and accepted, but has not been through the composition and copyediting processes. The final version may differ slightly in style or formatting and will contain links to any supplemental data.

Alerts: Sign up at rc.rcjournal.com/alerts to receive customized email alerts when the fully formatted version of this article is published. 
Effect of Prone Positioning on Clinical Outcomes of Non-Intubated Subjects with COVID-19: A Comparative Systematic Review and Meta-Analysis

Azizullah Beran; MD¹, Mohammed Mhanna; MD¹, Omar Srour; MD1․ Hazem, Ayesh; MD¹, Omar Sajdeya; MD ${ }^{1}$, Sami Ghazaleh; MD¹, Asmaa S. Mhanna; MD², Dana Ghazaleh; MD³, Waleed Khokher, MD¹, Aadil Maqsood; MD, Ragheb Assaly; $\mathrm{MD}^{4}$

${ }^{1}$ Department of Internal Medicine, University of Toledo, Toledo, OH, USA.

${ }^{2}$ University of Toledo, Toledo, OH, USA.

${ }^{3}$ University of Minnesota, Minneapolis, MN, USA.

${ }^{4}$ Department of Pulmonary and Critical Care Medicine, University of Toledo, Toledo, OH, USA.

Corresponding author: Azizullah Beran, M.D., Department of Internal Medicine, University of Toledo, 2100 W. Central Ave, Toledo, OH 43606, Tel: 469.348.1347, Email: Azizullah.Beran@utoledo.edu

Conflicts of interest: There are no conflicts of interest to disclose.

Funding: None.

Word count: 2459 .

Keywords: awake prone positioning, intubation, mortality, ICU admission, COVID-19, SARS-CoV-2.

Author contributions: Azizullah Beran: study design; data acquisition and interpretation, statistical analysis; manuscript drafting; statistical analysis. Mohammed Mhanna, Omar Srour, Hazem Ayesh, Omar Sajdeya, Sami Ghazaleh, Asmaa S. Mhanna, Dana Ghazaleh, Waleed Khokher, and Aadil Maqsood: data acquisition and interpretation; manuscript drafting. Ragheb Assaly: study supervision; critical revision for intellectual content. 
Data availability statement: The authors declare that all the data supporting the findings of this study are available within the manuscript. 


\section{Abstract}

Introduction: Awake Prone positioning (APP) has been recently proposed as an adjunctive treatment for nonintubated coronavirus disease 2019 (COVID-19) patients requiring oxygen therapy to improve oxygenation and reduce the risk of intubation. However, the magnitude of the effect of APP on clinical outcomes in these patients remains uncertain. We performed a comparative systematic review and meta-analysis to evaluate the effectiveness of APP to improve the clinical outcomes in non-intubated subjects with COVID-19.

Methods: The primary outcomes were the need for endotracheal intubation and mortality. The secondary outcome was the length of hospital stay. Pooled risk ratio (RR) and mean difference (MD) with the corresponding $95 \%$ confidence intervals (CIs) were obtained by the Mantel-Haenszel method within a random-effect model.

Results: A total of fourteen studies (five randomized controlled trials [RCT] and nine observational studies) involving 3324 subjects (1495 received APP and 1829 did not) were included. There was a significant reduction in the mortality rate in APP group compared to control ( $\mathrm{RR} 0.68,95 \%$ CI 0.51-0.90, $\mathrm{P}=0.008, \mathrm{I}^{2}=52 \%$ ) with no significant effect on intubation (RR $0.85,95 \%$ CI $0.66-1.08, \mathrm{P}=0.17, \mathrm{I}^{2}=63 \%$ ) or length of hospital stay (MD -3.09 days, $95 \% \mathrm{CI}$ $\left.10.14,3.96, \mathrm{P}=0.39, \mathrm{I}^{2}=97 \%\right)$. Subgroup analysis of $\mathrm{RCTs}$ showed significant reduction in intubation rate (RR 0.83 , $95 \%$ CI $0.72-0.97, \mathrm{P}=0.02, \mathrm{I}^{2}=0 \%$ ).

Conclusions: APP has the potential to reduce the in-hospital mortality rate in COVID-19 subjects with hypoxia without a significant effect on the need for intubation or length of hospital stay. However, there was a significant decrease in the need for intubation on subgroup analysis of RCTs. More large-scale trials with a standardized protocol for prone positioning are needed to better evaluate its effectiveness in this select population. 


\section{Introduction:}

Coronavirus disease 2019 (COVID-19) due to severe acute respiratory syndrome coronavirus 2 (SARS-CoV2) has led to significant morbidity and mortality globally ${ }^{1}$. Acute respiratory distress syndrome (ARDS) occurs in 20$41 \%$ of patients with severe COVID-19 2 . Prone positioning is known to improve oxygenation and mortality in mechanically ventilated and intubated patients with moderate-to-severe ARDS ${ }^{3}$. Prone positioning improves oxygenation by reducing ventilation-perfusion (V/Q) mismatch and reducing the intrapulmonary shunt ${ }^{4}$.

Utilizing awake prone positioning (APP) has been recently proposed as an adjunctive treatment for spontaneously breathing non-intubated COVID-19 patients requiring oxygen therapy to reduce the risk of intubation 5. Most published studies showed significant improvement in oxygenation parameters such as the ratio of arterial oxygen partial pressure to fractional inspired oxygen ( $\mathrm{PaO} 2 / \mathrm{FiO} 2), \mathrm{PaO} 2$, and oxygen saturation $(\mathrm{SpO} 2)$ after APP sessions ${ }^{4-6}$. Several single-arm meta-analyses have evaluated the effect of APP on oxygenation parameters and pooled the overall mortality and intubation rates in subjects who underwent APP without a control group ${ }^{7}$ However, the magnitude of the effect of APP on clinical outcomes (e.g., the risk of endotracheal intubation or mortality) in subjects with COVID-19 remains uncertain. Therefore, we performed a comparative meta-analysis to evaluate the effectiveness of APP to improve the clinical outcomes in subjects with COVID-19.

\section{Methods:}

\section{Data sources and search strategy}

We performed a comprehensive search for published studies indexed in PubMed/MEDLINE, EMBASE, and the Cochrane Central Register of Controlled Trials from inception to August 30, 2021. We also performed a manual search for additional relevant studies using references of the included articles. The following search terms were used: ("prone positioning" or "prone position") and (“COVID-19" or "SARS-CoV-2")". The search was not limited by language, study design, or country of origin. Supplementary Table 1 describes the full search term used in each database searched. 


\section{Inclusion and exclusion criteria}

All published studies (randomized controlled trials (RCTs) and observational studies) that compared APP versus the control group in non-intubated COVID-19 subjects and reported one of the following outcomes: endotracheal intubation, mortality, and length of hospital stay were eligible for inclusion. All the studies that did not report endotracheal intubation or mortality rates were excluded, such as a study by Kharat et al. ${ }^{8}$ Only adult subjects aged 18 years or older were eligible for inclusion. We excluded single-arm studies, case reports, reviews, commentaries, preprints (not peer-reviewed), and abstracts.

\section{Data extraction}

The following data were extracted from the studies: first author name, publication year, country of origin, study design, number of subjects, follow-up duration, subject location, outcomes measures including rates of intubation, mortality, and length of hospital stay. We also extracted details of APP, oxygen and non-invasive respiratory support, and subjects' baseline comorbidities, including body mass index (BMI), hypertension, diabetes mellitus, and chronic obstructive lung disease (COPD). We followed the Preferred Reporting Items for Systematic Reviews and Meta-Analyses (PRISMA) Statement guidelines to select the final studies. Two investigators (MM and $\mathrm{AB})$ independently performed the search and shortlisted the studies for final review. Discrepancies were resolved by a third reviewer (OS). We did not contact the authors of the studies.

\section{Outcomes}

The primary outcomes of our study were the need for endotracheal intubation and mortality between the APP and control groups of non-intubated subjects with COVID-19. The secondary outcome was the length of hospital stay between the two groups.

\section{Statistical analysis}

We performed a meta-analysis of the included studies using Review Manager 5.3 (Cochrane Collaboration, Copenhagen, The Nordic Cochrane Centre) and Comprehensive Meta-Analysis (Biostat, Englewood, USA). The median and interquartile range were converted to mean and SD where applicable ${ }^{9}$. The random-effects model was used to calculate the pooled risk ratio (RR) and mean difference (MD) with the corresponding confidence intervals (CI) for proportional and continuous variables, respectively. A $P$-value $<0.05$ was considered statistically significant. 
The heterogeneity of the effect size estimates across the studies was quantified using the Q statistic and $\mathrm{I}^{2}(\mathrm{P}<0.10$ was considered significant). A value of $\mathrm{I}^{2}$ of $0-25 \%$ indicates insignificant heterogeneity, $26-50 \%$ low heterogeneity, $51-75 \%$ moderate heterogeneity, and $76-100 \%$ high heterogeneity ${ }^{10}$.

\section{Subgroup and sensitivity analyses for endotracheal intubation and mortality}

We performed a subgroup analysis of RCTs for endotracheal intubation. To confirm the robustness of the results, sensitivity analysis for endotracheal intubation and mortality using leave-one-out meta-analysis was performed to see if it had a significant influence on the result of the meta-analysis.

\section{Bias assessment}

The Jadad composite scale was used to assess the methodological quality of the RCTs based on randomization, blinding, and withdrawals ${ }^{11}$. The scale ranged from 0 to 5 points ${ }^{12}$. Studies with a total score $>3$ were considered high quality, while moderate quality if a score of 3 and low quality if $<3$. The Newcastle Ottawa Quality Assessment Scale (NOS) was used to assess the quality of the observational studies based on the selection of the study groups, comparability of study groups, and ascertainment of exposure/outcome ${ }^{12}$. Studies with a total score $>6$ were considered high quality, while moderate quality if a score of 6 and low quality if $<6$. Publication bias was assessed qualitatively by visually assessing the funnel plot and quantitively using Egger's regression analysis. Two authors (AB and $\mathrm{MM})$ independently assessed each study for bias. Discrepancies were resolved by a third reviewer (OS).

\section{Results:}

\section{Study selection}

A total of 1048 studies were retrieved by our search strategy. Among these, 57 were eligible for systematic review. Subsequently, we excluded 44 studies because of the following: subjects were intubated, absence of control group, lack of appropriate outcome, or lack of appropriate control. Eventually, 14 studies ${ }^{5,6,13-24}$ met our inclusion criteria and were included in the meta-analysis. Figure 1 shows the PRISMA flow chart that illustrates how the final studies were selected. 


\section{Quality and publication bias assessment}

Quality assessment scores of the RCTs and observational studies are summarized in Supplementary table 2. All the included studies were of moderate or high quality. Four $\operatorname{RCTs}^{6,20,22,23}$ were of moderate quality, and one $\mathrm{RCT}^{17}$ was of high quality. Six observational studies ${ }^{5,13,15,16,19,21}$ had a total score $>6$, indicating that they were of high quality. Three observational studies ${ }^{14,18,24}$ scored 6 , indicating that they were of moderate quality.

There was a visible asymmetry in the funnel plot of the studies that reported both the need for endotracheal intubation and in-hospital mortality, which may suggest the presence of publication bias (Figure 4). However, Egger's regression analysis did not demonstrate statistically significant publication bias $(\mathrm{P}=0.54$ and $\mathrm{P}=0.91$ for endotracheal intubation and mortality, respectively).

\section{Study and subjects' characteristics}

Tables 1 and 2 show the study and subject characteristics of the studies included in the meta-analysis. All the included studies were published between 2020 and 2021 and included spontaneously breathing non-intubated subjects with laboratory-confirmed COVID-19. Based on country of origin, three studies originated from the United States; five studies originated from Europe (Italy, France, Spain, and Sweden), three studies from Asia (China and India), two studies from South America (Brazil, Mexico, and Ecuador), and one multinational study (meta-trial from 6 countries: Canada, Ireland, France, Mexico, Spain, and USA). Regarding the design of included studies, five were RCTs and nine were observational cohort studies (six studies were retrospective cohort, and three were prospective cohort).

A total of 3324 subjects (1495 received APP and 1829 did not) were included, with males representing 69.8\% of the total subjects. The follow-up period across the studies ranged from 14 to 90 days. The assessment of the risk of bias is presented in Supplementary table 2 . All nine observational studies scored $\geq 6$ on the Newcastle-Ottawa Scale, and all five RCTs scored $\geq 3$, representing a low risk of bias (Supplementary table 2).

\section{Outcomes}

Need for endotracheal intubation 
Table 2 summarizes the outcomes of the individual studies included in the meta-analysis. Across the 13 studies $^{5,6,13-20,22-24}$ that reported the intubation rate, 27\% of subjects who received APP required intubation compared to $29.8 \%$ in subjects who did not receive APP. The need for endotracheal intubation was similar between APP and control groups (RR $0.85,95 \%$ CI $0.66-1.08, \mathrm{P}=0.17$ ), the statistical heterogeneity was moderate with $\mathrm{I}^{2}$ of $63 \%$ (Figure 2A). However, on subgroup analysis of RCTs, the need for intubation significantly reduced in APP group versus control (RR $0.83,95 \%$ CI $0.72-0.97, \mathrm{P}=0.02, \mathrm{I}^{2}=0 \%$ ) (Figure 3 ). To assess the stability of the results of our meta-analyses, we performed a 1-study removed sensitivity analysis. Removal of Zang et al. moved the overall effect to favor APP with an RR of 0.80 (95\% CI: 0.64-0.99), suggesting that Zang et al. was partly the reason for the moderate between-study heterogeneity. (Supplementary figure 1).

\section{Mortality}

Thirteen studies 5 , 6, 13, 14, 16-24 reported the mortality rate. The mortality rate was $17.9 \%$ in the APP group compared to $25.7 \%$ in the control group. There was a statistically significant difference in the mortality rate between the two groups (RR $0.68,95 \%$ CI $0.51-0.90, \mathrm{P}=0.008)$ and the statistical heterogeneity was moderate with $\mathrm{I}^{2}$ of $52 \%$ (Figure 2B). A leave-one-out sensitivity analysis showed consistent results (Supplementary figure 2).

\section{Length of hospital stay}

Six studies ${ }^{6,14,16,17,19,23}$ reported the length of hospital stay. There was no significant difference with regards to the length of hospital stay (MD -3.09 days, 95\% CI -10.14, 3.96, $\mathrm{P}=0.39, \mathrm{I}^{2}=97 \%$ ) (Figure 2C).

\section{Discussion:}

Prone positioning for treating ARDS is a well-known strategy to improve oxygenation; it is recommended for 16 hours daily in mechanically ventilated and intubated subjects with ARDS with PaO2/FiO2 ratio less than 150 $\mathrm{mmHg}{ }^{3}$. However, the evidence regarding the utility of APP in non-intubated subjects is limited.

In the current COVID-19 pandemic era, a substantial number of COVID-19 patients developed ARDS with an extreme surge in need for respiratory support, intubation, and mechanical ventilation. Hence, every effort was implemented to avoid intubation and mechanical ventilation, given the shortage of human and medical resources ${ }^{25}$. 
One of the proposed strategies to avoid such complications is to implement prone positioning in awake, spontaneously breathing patients in the hope of preventing further respiratory deterioration and the need for advanced respiratory support ${ }^{26}$.

Several studies have shown significant improvement in oxygenation parameters such as $\mathrm{PaO} 2 / \mathrm{FiO} 2, \mathrm{PaO} 2$, $\mathrm{SpO} 2$, and respiratory rate ${ }^{27-29}$. Some meta-analyses have investigated the effect of APP on clinical outcomes in nonintubated patients with COVID-19 by pooling the rates of intubation and mortality from single-arm studies which did not have a control group ${ }^{7,30}$. A meta-analysis by Pavlov et al..$^{30}$ has pooled the mortality and intubation rates in singlearm studies and showed the pooled intubation rate of $27 \%$ among those who underwent APP. However, few studies have evaluated the effects of APP vs. control on clinical outcomes of patients with COVID-1913, 14, 18, 19. To our knowledge, only one comparative meta-analysis by Chua et al. ${ }^{31}$ has been published that evaluated the impact of APP vs. control on intubation and mortality rates in subjects with COVID-19. However, Chua et al. ${ }^{31}$ included a limited number of studies (five studies), and all the included studies were observational (no RCT). Further studies, including $\operatorname{RCTs}^{6,20,23}$ have been published, and we provide an updated comparative meta-analysis to investigate the effect of APP compared to control on the clinical outcomes, including the need for intubation, in-hospital mortality, and length of hospital stay.

Our meta-analysis demonstrated that APP reduced mortality in non-intubated COVID-19 subjects without a significant difference in the need for endotracheal intubation and length of hospital stay. However, APP showed a significant reduction in the need for intubation when subgroup analysis was restricted to RCTs.

Our meta-analysis results are similar to those from the recent meta-analysis by Chua et al. ${ }^{31}$ which revealed that APP could decrease mortality rate (Odds ratio $(\mathrm{OR}): 0.35,95 \% \mathrm{CI}: 0.16-0.75, \mathrm{P}=0.007$ ) without significant effect on intubation rate (OR: 1.20, 95\% CI: 0.77-1.86, $\mathrm{P}=0.42)$ in non-intubated COVID-19 patients with hypoxia. However, we further conducted a subgroup analysis of RCTs for intubation, which significantly reduced the need for intubation. Furthermore, sensitivity analysis did not show consistent findings for intubation. This discrepancy might be attributed to the inconsistent protocols of APP utilized in the included studies and to the absence of standardized criteria and indications for intubation ${ }^{32}$. Thus, we believe that there is an urgent need for guidelines and protocols to guide the practice of APP in COVID-19 subjects. The protocols should include the eligibility criteria to initiate APP, 
number of sessions and average time per day, proper follow-up protocols, early identification of complications and treatment failure, and standardized criteria for intubation.

Our study has certain limitations. First, this meta-analysis was mainly based on observational studies and only included five RCTs. Therefore, more large-scale RCTs to investigate the impact of APP on the clinical outcomes of COVID-19 subjects are warranted. Second, moderate heterogeneity was found in the measurement of intubation and mortality. This can be explained by inconsistent follow-up duration among the studies, significant variation in the used respiratory supports, and wide range of frequency and duration of the applied APP protocols, and significant difference in the location of the study (ED vs. hospital ward vs. ICU) ${ }^{32}$. Third, we were not able to assess the oxygenation parameters before and after APP sessions due to limited reported data. Fourth, the majority of the studies did not assess the APP protocol adherence, and there was a lack of standardized protocol and optimal duration for APP among studies. Lastly, the lack of patient-level data did not allow to control for possible variations in baseline characteristics or adoption of APP.

Despite the limitations, our study has significant strengths. First, we included a total of 14 studies with over 3300 subjects with COVID-19. This is by far the largest analysis comparing the effect of APP on clinical outcomes in COVID-19 subjects with hypoxia. Although significant heterogeneity was noted, we performed a subgroup analysis of RCTs and sensitivity analysis to evaluate the robustness of our results. Consistent results were observed on sensitivity analysis for morality. Despite the presumed heterogeneity, the meta-analysis was undertaken to observe the effect of APP in subjects with COVID-19, as several studies revealed some benefits in terms of mortality and intubation rates. Finally, all the included studies in our study were of moderate or high quality based on quality assessment.

In conclusion, APP has the potential to reduce the in-hospital mortality rate in COVID-19 subjects with hypoxia without a significant effect on the need for intubation or length of hospital stay. However, there was a significant decrease in the need for intubation on subgroup analysis of RCTs. More large-scale trials with a standardized protocol for APP are needed to better evaluate its effectiveness in this select population. 


\section{References:}

1. Kiang MV, Irizarry RA, Buckee CO, Balsari S. Every Body Counts: Measuring Mortality From the COVID-19 Pandemic. Ann Intern Med 2020;173(12):1004-1007.

2. Knoebl P, Cataland S, Peyvandi F, Coppo P, Scully M, Kremer Hovinga JA, et al. Efficacy and safety of open-label caplacizumab in patients with exacerbations of acquired thrombotic thrombocytopenic purpura in the HERCULES study. J Thromb Haemost 2020;18(2):479-484.

3. Guérin C, Reignier J, Richard JC, Beuret P, Gacouin A, Boulain T, et al. Prone positioning in severe acute respiratory distress syndrome. N Engl J Med 2013;368(23):2159-2168.

4. Singh P, Jain P, Deewan H. Awake Prone Positioning in COVID-19 Patients. Indian J Crit Care Med 2020;24(10):914-918.

5. Ferrando C, Mellado-Artigas R, Gea A, Arruti E, Aldecoa C, Adalia R, et al. Awake prone positioning does not reduce the risk of intubation in COVID-19 treated with high-flow nasal oxygen therapy: a multicenter, adjusted cohort study. Crit Care 2020;24(1):597.

6. Johnson SA, Horton DJ, Fuller MJ, Yee J, Aliyev N, Boltax JP, et al. Patient-Directed Prone Positioning in Awake Patients with COVID-19 Requiring Hospitalization (PAPR). Ann Am Thorac Soc 2021.

7. Cardona S, Downing J, Alfalasi R, Bzhilyanskaya V, Milzman D, Rehan M, et al. Intubation rate of patients with hypoxia due to COVID-19 treated with awake proning: A meta-analysis. Am J Emerg Med 2021;43:88-96.

8. Kharat A, Dupuis-Lozeron E, Cantero C, Marti C, Grosgurin O, Lolachi S, et al. Self-Proning in COVID-19 Patients on Low-Flow Oxygen Therapy. A Cluster Randomised Controlled Trial. ERJ Open Res 2021:00692-02020.

9. Luo D, Wan X, Liu J, Tong T. Optimally estimating the sample mean from the sample size, median, mid-range, and/or mid-quartile range. Stat Methods Med Res 2018;27(6):1785-1805.

10. Higgins JP, Thompson SG, Deeks JJ, Altman DG. Measuring inconsistency in meta-analyses. Bmj 2003;327(7414):557-560.

11. Moher D, Pham B, Jones A, Cook DJ, Jadad AR, Moher M, et al. Does quality of reports of randomised trials affect estimates of intervention efficacy reported in meta-analyses? Lancet 1998;352(9128):609-613.

12. Stang A. Critical evaluation of the Newcastle-Ottawa scale for the assessment of the quality of nonrandomized studies in meta-analyses. Eur J Epidemiol 2010;25(9):603-605.

13. Prud'homme E, Trigui Y, Elharrar X, Gaune M, Loundou A, Lehingue S, et al. Effect of Prone Positioning on the Respiratory Support of Nonintubated Patients With Coronavirus Disease 2019 and Acute Hypoxemic Respiratory Failure: A Retrospective Matching Cohort Study. Chest 2021.

14. Jagan N, Morrow LE, Walters RW, Klein LP, Wallen TJ, Chung J, et al. The POSITIONED Study: Prone Positioning in Nonventilated Coronavirus Disease 2019 Patients-A Retrospective Analysis. Crit Care Explor 2020;2(10):e0229.

15. Mansueto G, Gatti FL, Boninsegna E, Conci S, Guglielmi A, Contro A. Biliary Leakage After Hepatobiliary and Pancreatic Surgery: A Classification System to Guide the Proper Percutaneous Treatment. Cardiovasc Intervent Radiol 2020;43(2):302-310. 
16. Tonelli R, Pisani L, Tabbì L, Comellini V, Prediletto I, Fantini R, et al. Early awake proning in critical and severe COVID-19 patients undergoing noninvasive respiratory support: $A$ retrospective multicenter cohort study. Pulmoe 2021.

17. Taylor SP, Bundy H, Smith WM, Skavroneck S, Taylor B, Kowalkowski MA. Awake-Prone Positioning Strategy for Non-Intubated Hypoxic Patients with COVID-19: A Pilot Trial with Embedded Implementation Evaluation. Ann Am Thorac Soc 2020.

18. Padrão EMH, Valente FS, Besen B, Rahhal H, Mesquita PS, de Alencar JCG, et al. Awake Prone Positioning in COVID-19 Hypoxemic Respiratory Failure: Exploratory Findings in a Single-center Retrospective Cohort Study. Acad Emerg Med 2020;27(12):1249-1259.

19. Zang X, Wang Q, Zhou H, Liu S, Xue X, Group C-EPPS. Efficacy of early prone position for COVID19 patients with severe hypoxia: a single-center prospective cohort study. Intensive Care Med 2020;46(10):1927-1929.

20. Jayakumar D, Ramachandran Dnb P, Rabindrarajan Dnb E, Vijayaraghavan Md BKT, Ramakrishnan Ab N, Venkataraman Ab R. Standard Care Versus Awake Prone Position in Adult Nonintubated Patients With Acute Hypoxemic Respiratory Failure Secondary to COVID-19 Infection-A Multicenter Feasibility Randomized Controlled Trial. J Intensive Care Med 2021;36(8):918-924.

21. Jouffroy R, Darmon M, Isnard F, Geri G, Beurton A, Fartoukh M, et al. Impact of prone position in non-intubated spontaneously breathing patients admitted to the ICU for severe acute respiratory failure due to COVID-19. J Crit Care 2021;64:199-204.

22. Rosén J, von Oelreich E, Fors D, Jonsson Fagerlund M, Taxbro K, Skorup P, et al. Awake prone positioning in patients with hypoxemic respiratory failure due to COVID-19: the PROFLO multicenter randomized clinical trial. Crit Care 2021;25(1):209.

23. Ehrmann S, Li J, Ibarra-Estrada M, Perez Y, Pavlov I, McNicholas B, et al. Awake prone positioning for COVID-19 acute hypoxaemic respiratory failure: a randomised, controlled, multinational, open-label meta-trial. Lancet Respir Med 2021:S2213-2600(2221)00356-00358.

24. Perez-Nieto OR, Escarraman-Martinez D, Guerrero-Gutierrez MA, Zamarron-Lopez El, MancillaGalindo J, Kammar-García A, et al. Awake prone positioning and oxygen therapy in patients with COVID-19: The APRONOX study. Eur Respir J 2021:2100265.

25. Wunsch H. Mechanical Ventilation in COVID-19: Interpreting the Current Epidemiology. Am J Respir Crit Care Med 2020;202(1):1-4.

26. Sun Q, Qiu H, Huang M, Yang Y. Lower mortality of COVID-19 by early recognition and intervention: experience from Jiangsu Province. Annals of intensive care 2020;10(1):1-4.

27. Tan W, Xu DY, Xu MJ, Wang ZF, Dai B, Li LL, et al. The efficacy and tolerance of prone positioning in non-intubation patients with acute hypoxemic respiratory failure and ARDS: a meta-analysis. Ther Adv Respir Dis 2021;15:17534666211009407.

28. Behesht Aeen F, Pakzad R, Goudarzi Rad M, Abdi F, Zaheri F, Mirzadeh N. Effect of prone position on respiratory parameters, intubation and death rate in COVID-19 patients: systematic review and meta-analysis. Sci Rep 2021;11(1):14407.

29. Sartini C, Tresoldi M, Scarpellini P, Tettamanti A, Carcò F, Landoni G, et al. Respiratory Parameters in Patients With COVID-19 After Using Noninvasive Ventilation in the Prone Position Outside the Intensive Care Unit. JAMA 2020;323(22):2338-2340.

30. Pavlov I, He H, McNicholas B, Perez Y, Tavernier E, Trump MW, et al. Awake prone positioning in non-intubated patients with acute hypoxemic respiratory failure due to COVID-19: A systematic review of proportional outcomes comparing observational studies with and without awake prone positioning in the setting of COVID-19. Respir Care 2021:respcare.09191. 
31. Chua EX, Zahir SMISM, Ng KT, Teoh WY, Hasan MS, Ruslan SRB, et al. Effect of prone versus supine position in COVID-19 patients: A systematic review and meta-analysis. J Clin Anesth 2021;74:110406-110406.

32. Ponnapa Reddy M, Subramaniam A, Afroz A, Billah B, Lim ZJ, Zubarev A, et al. Prone Positioning of Nonintubated Patients With Coronavirus Disease 2019-A Systematic Review and MetaAnalysis. Crit Care Med 2021;49(10):e1001-e1014.

Figure legends:

Figure 1: PRISMA flow diagram for the selection of studies.

Figure 2: Forest plots comparing awake prone positioning and control regarding: (A) endotracheal intubation, (B) mortality, and (C) length of hospital stay.

Figure 3: Subgroup analysis of RCTs for the need for endotracheal intubation.

Figure 4: Funnel plots comparing awake prone positioning and control regarding: (A) endotracheal intubation and (B) mortality. 


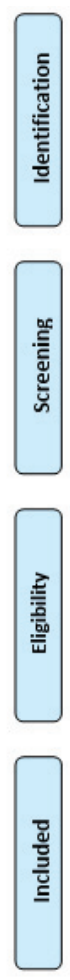

Records identified through PubMed/MEDLINE database

$$
(n=341)
$$

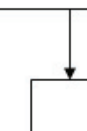

Records after duplicates removed $(n=797)$
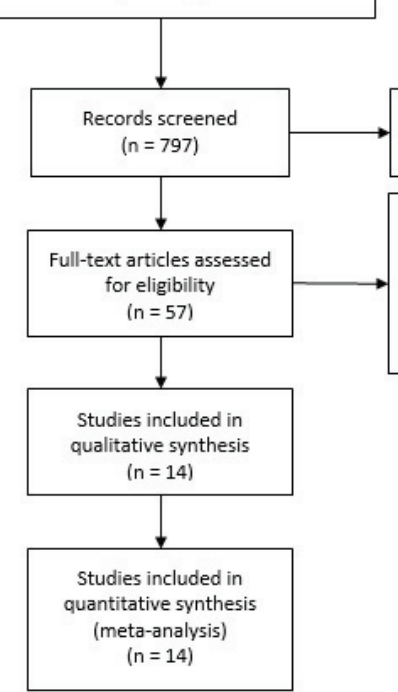

Records identified through othe databases: Embase $(n=636)$ Cochrane $(n=71)$
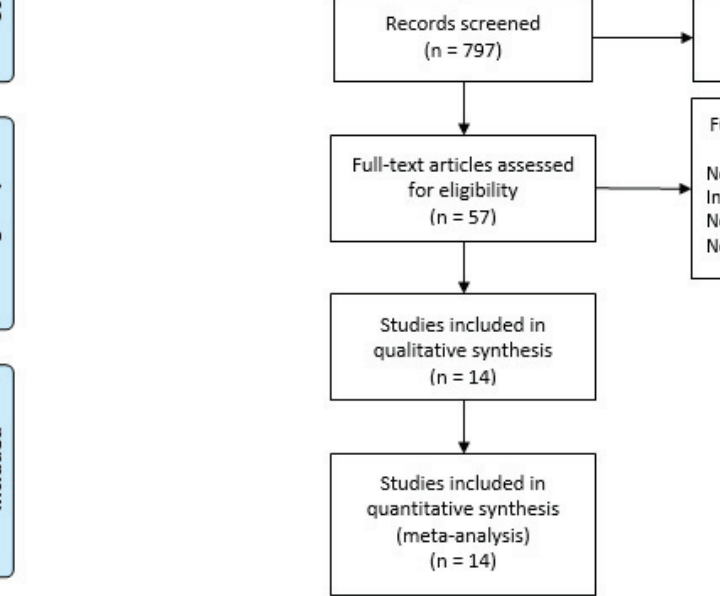

Records excluded ( $n=3176$ )

Full-text articles excluded ( $n=43$ )

No control group $(n=29)$ ntubated patients $(n=7)$

No appropriate control $(n=4)$

No appropriate outcome $(n=3)$

Figure 1: PRISMA flow diagram for the selection of studies.

$432 \times 396 \mathrm{~mm}(38 \times 38 \mathrm{DPI})$ 


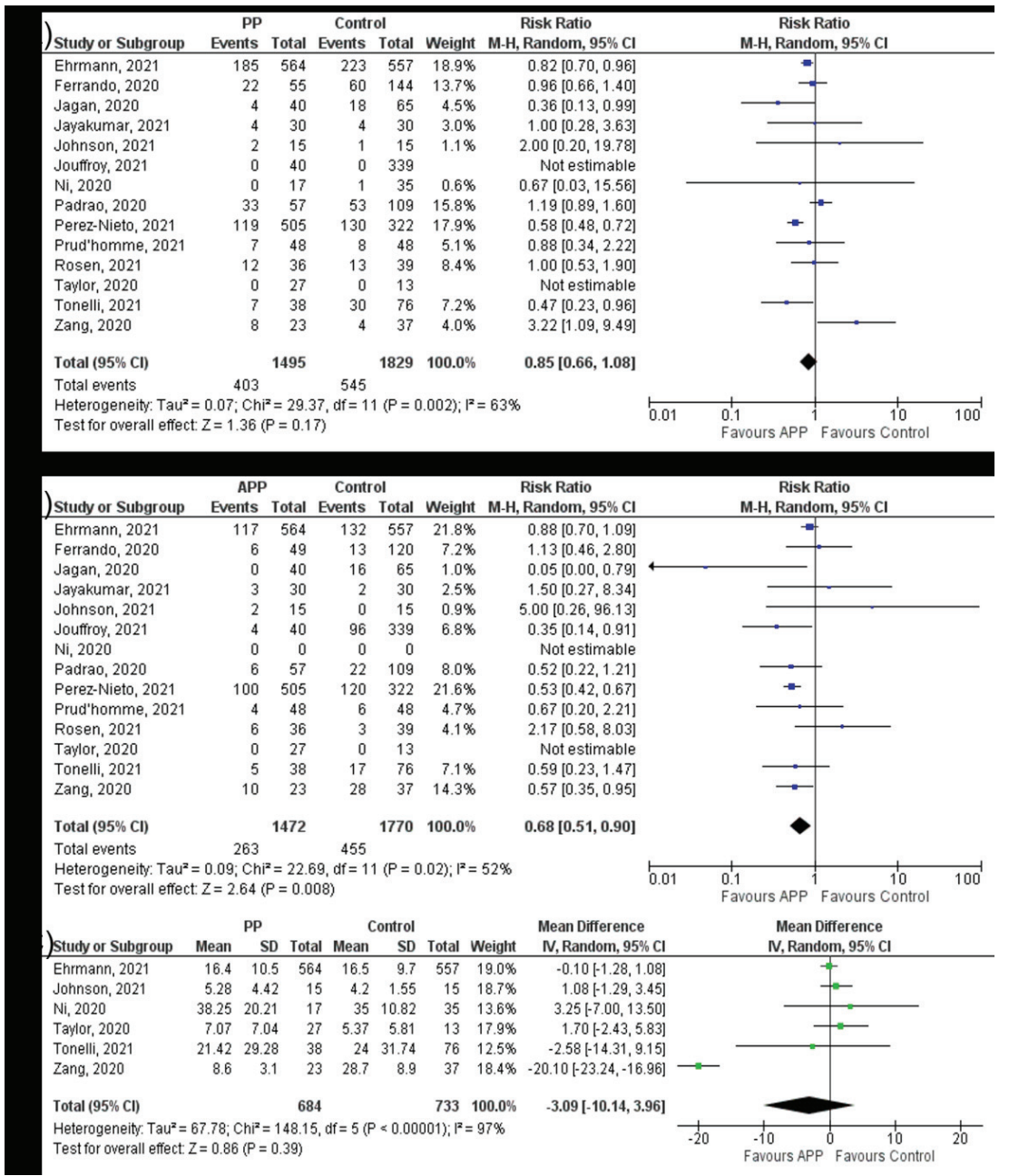

Forest plots comparing awake prone positioning and control regarding: (A) endotracheal intubation, (B) mortality, and (C) length of hospital stay. 


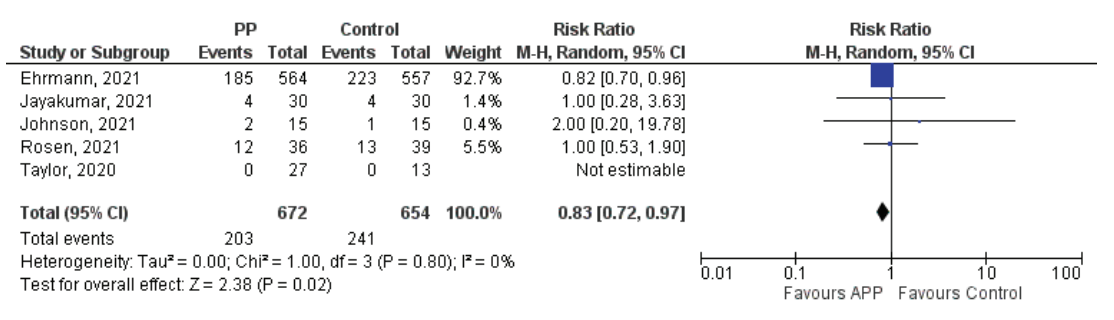

Subgroup analysis of RCTs for the need for endotracheal intubation.

$268 \times 73 \mathrm{~mm}(72 \times 72 \mathrm{DPI})$ 


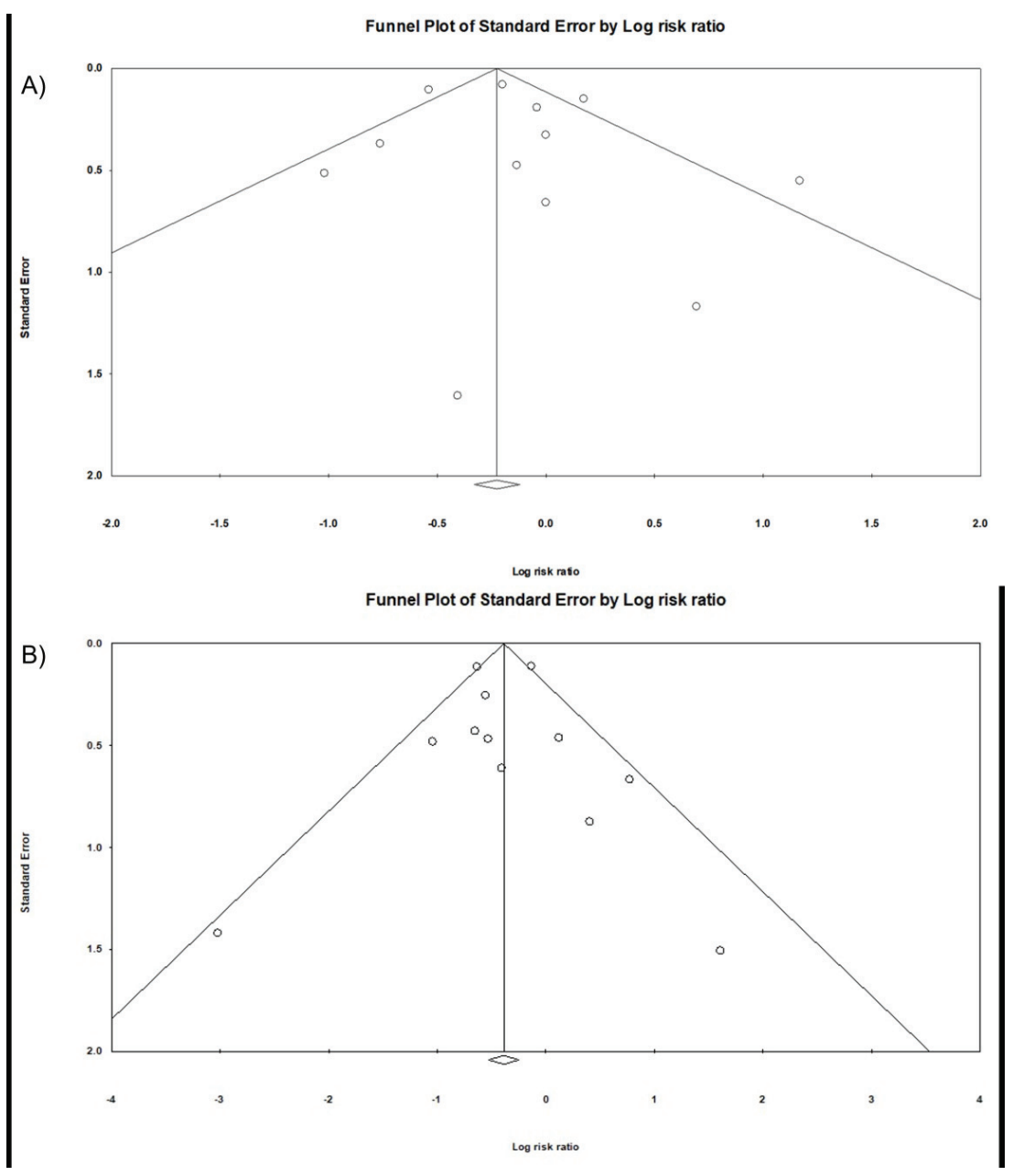

Figure 4: Funnel plots comparing awake prone positioning and control regarding: $(A)$ endotracheal intubation and (B) mortality.

$651 \times 751 \mathrm{~mm}(130 \times 130 \mathrm{DPI})$ 
Table 1: Study characteristics of the included studies.

\begin{tabular}{|c|c|c|c|c|c|c|c|c|c|}
\hline Study, year & Study design & Country & $\begin{array}{l}\text { Total sample } \\
\text { size } \\
\text { (APP/Control), } \\
\text { n }\end{array}$ & $\begin{array}{l}\text { Male, n } \\
(\%)\end{array}$ & $\begin{array}{l}\text { Age, } \\
\text { mean } \pm S D, \\
\text { years }\end{array}$ & $\begin{array}{l}\text { Subject } \\
\text { location of } \\
\text { APP }\end{array}$ & $\begin{array}{l}\text { Oxygen \& non- } \\
\text { invasive } \\
\text { respiratory } \\
\text { support }\end{array}$ & Details of APP & $\begin{array}{l}\text { Follow- } \\
\text { up period }\end{array}$ \\
\hline $\begin{array}{l}\text { Ehrmann, } \\
2021^{23}\end{array}$ & RCT & Multinational & $1121(564 / 557)$ & $746(66.5)$ & $61.1 \pm 13.7$ & Ward \& ICU & HFNC & $\begin{array}{l}151 \text { patients had } \geq 8 \text { hours } \\
\text { of APP daily }\end{array}$ & 28 days \\
\hline Ferrando, $2020^{5}$ & $\mathrm{PC}$ & Spain & $199(55 / 144)$ & $147(73.9)$ & $62.6 \pm 12.04$ & $\mathrm{ICU}$ & HFNC & $\begin{array}{l}>16 \text { hour/day regardless } \\
\text { of APP session numbers }\end{array}$ & 28 days \\
\hline Jagan, 2020 ${ }^{14}$ & $\mathrm{RC}$ & USA & $105(40 / 65)$ & $57(54.3)$ & $62.1 \pm 16.3$ & Ward & NR & $\begin{array}{l}\geq 1 \text { hour for } \geq 5 \text { occasions } \\
\text { per day and } \geq 1 \text { hour } \\
\text { overnight }\end{array}$ & NR \\
\hline $\begin{array}{l}\text { Jayakumar, } \\
2021^{20}\end{array}$ & RCT & India & $60(30 / 30)$ & $50(83.3)$ & $56.1 \pm 11.6$ & ICU & $\begin{array}{l}\text { SFM, NRM, HFNC, } \\
\text { NIV }\end{array}$ & $\begin{array}{l}13 \quad(43 \%) \quad \text { patients } \\
\text { completed mean of } \geq 6 \\
\text { hours daily in APP }\end{array}$ & NR \\
\hline Johnson, 2021 ${ }^{6}$ & RCT & USA & $30(15 / 15)$ & $16(53.3)$ & $57.2 \pm 19.5$ & Ward & $\mathrm{NC}$ & $\begin{array}{l}\text { Average } 1.6 \text { hours in the } \\
\text { first } 72 \text { hours }\end{array}$ & NR \\
\hline Jouffroy, $2021^{21}$ & $\mathrm{RC}$ & France & $379(40 / 339)$ & $291(76.8)$ & $59(56-64)^{*}$ & ICU & $\begin{array}{l}\text { NIV, CPAP, HFNC, } \\
\text { NC, SFM }\end{array}$ & $\begin{array}{l}\text { Two 3-6-hour APP } \\
\text { sessions daily }\end{array}$ & 28 days \\
\hline
\end{tabular}




\begin{tabular}{|c|c|c|c|c|c|c|c|c|c|}
\hline $\mathrm{Ni}, \mathbf{2 0 2 0}^{15}$ & PC & China & $52(17 / 35)$ & $32(61.5)$ & $62 \pm 12$ & Ward & $\begin{array}{l}\text { HFNC, NC, mask, } \\
\text { NIV }\end{array}$ & $\begin{array}{l}\geq 4 \text { hours per day for } 10 \\
\text { days }\end{array}$ & 14 days \\
\hline Padrao, 2020 ${ }^{18}$ & $\mathrm{RC}$ & Brazil & $166(57 / 109)$ & $112(67.5)$ & $58.1 \pm 14.1$ & ED & $\begin{array}{l}\mathrm{NC} \text {, venturi mask, } \\
\mathrm{NRM}\end{array}$ & $\begin{array}{l}58 \% \text { had }>4 \text { hours per } \\
\text { session, } 2 \text { APP sessions } \\
\text { per day }\end{array}$ & 15 days \\
\hline $\begin{array}{l}\text { Perez-Nieto, } \\
2021^{24}\end{array}$ & $\mathrm{RC}$ & $\begin{array}{l}\text { Mexico and } \\
\text { Ecuador }\end{array}$ & $827(505 / 322)$ & $600(72.6)$ & $54.3(14.2)$ & Ward \& ICU & $\begin{array}{l}\text { LFNC, HFNC, } \\
\text { NRM }\end{array}$ & $\begin{array}{l}\text { Median APP time was } 12 \\
\text { (IQR: 8-24) }\end{array}$ & NR \\
\hline $\begin{array}{l}\text { Prud'homme, } \\
2021^{13}\end{array}$ & $\mathrm{RC}$ & France & $96(48 / 48)$ & $68(70.8)$ & $61.5 \pm 14.8$ & Ward & $\mathrm{NC}, \mathrm{HFNC}$ & $\begin{array}{l}\text { Mean } 6.9 \pm 5.2 \text { days }(32 \\
\text { patients proned for } 3-8 \\
\text { hours/day and } 16 \text { proned } \\
>8 \text { hours/day) }\end{array}$ & 14 days \\
\hline Rosen, $\mathbf{2 0 2 1}^{22}$ & RCT & Sweden & $75(36 / 39)$ & $55(73.3)$ & $63.7 \pm 14$ & Ward \& ICU & HFNC, NIV & $\begin{array}{l}\text { Median } 9 \text { hours daily } \\
(4.4-10.6)\end{array}$ & 30 days \\
\hline Taylor, 2020 ${ }^{17}$ & RCT & USA & $40(27 / 13)$ & $27(67.5)$ & $56.7 \pm 14.1$ & Ward & NC, MFNC, BiPAP & NR & 30 days \\
\hline Tonelli, 2021 ${ }^{16}$ & $\mathrm{RC}$ & Italy & $114(38 / 76)$ & $80(70.2)$ & $67(32-80)^{*}$ & $\mathrm{ICU}$ & HFNC, CPAP, NIV & $\begin{array}{l}\text { Varied from } 1 \text { to } 4 \\
\text { sessions daily }\end{array}$ & 30 days \\
\hline Zang, 2020 ${ }^{19}$ & $\mathrm{PC}$ & China & $60(30 / 30)$ & $39(65)$ & $64.8 \pm 9.9$ & NR & HFNC, NIV & $\begin{array}{l}\text { Mean } 13.4 \text { hours of APP } \\
\text { (range 6-30) }\end{array}$ & 90 days \\
\hline
\end{tabular}

Abbreviations: APP: awake prone positioning, BiPAP: bilevel positive airway pressure, CPAP: continuous positive airway pressure, HFNC, high-flow nasal cannula, ICU: intensive care unit, IQR: interquartile range, LFNC: low-flow nasal cannula, MFNC: median-flow nasal cannula, n: sample size, NC: nasal cannula, 
NIV: non-invasive ventilation, NRM: non-rebreather mask, NR: not reported, PC: prospective cohort, RCT: randomized controlled trial, RC: retrospective cohort, SD: standard deviation, SFM: simple face mask, USA: United States.

*Median (interquartile range).

Table 2: Subject characteristics and outcomes of the included studies in the meta-analysis.

\begin{tabular}{|c|c|c|c|c|c|c|c|c|c|c|c|c|c|c|}
\hline \multirow[t]{2}{*}{ Study, year } & \multicolumn{2}{|c|}{$\begin{array}{l}\text { BMI, median (IQR) or } \\
\operatorname{mean} \pm \mathrm{SD}, \mathrm{kg} / \mathrm{m}^{2}\end{array}$} & \multicolumn{2}{|c|}{ COPD, $n$} & \multicolumn{2}{|c|}{ HTN, n } & \multicolumn{2}{|l|}{ DM, n } & \multicolumn{2}{|c|}{ Intubation, $\mathbf{n}$} & \multicolumn{2}{|c|}{ Mortality, n } & \multicolumn{2}{|c|}{$\begin{array}{l}\text { Length of hospital stay, } \\
\text { median (IQR) or mean } \pm S D \text {, } \\
\text { days }\end{array}$} \\
\hline & APP & Control & APP & $\begin{array}{l}\text { Contro } \\
1\end{array}$ & APP & $\begin{array}{l}\text { Contro } \\
1\end{array}$ & APP & Control & APP & Control & APP & Control & APP & Control \\
\hline $\begin{array}{l}\text { Ehrmann, } \\
2021^{23}\end{array}$ & \multicolumn{2}{|c|}{ NR } & \multicolumn{2}{|c|}{ NR } & \multicolumn{2}{|c|}{ NR } & 176 & 173 & 185 & 223 & 117 & 132 & $16.4 \pm 10.5$ & $16.5 \pm 9.7$ \\
\hline $\begin{array}{l}\text { Ferrando, } \\
2020^{5}\end{array}$ & $\begin{array}{c}27.3(25.1- \\
29.4)\end{array}$ & $\begin{array}{c}26.8(24.8- \\
31.2)\end{array}$ & 4 & 6 & 20 & 60 & 9 & 23 & 22 & 60 & 6 & 13 & NR & \\
\hline Jagan, 2020 ${ }^{14}$ & $\begin{array}{c}31.3(26.4- \\
37.5)\end{array}$ & $\begin{array}{c}28(24.9- \\
34.4)\end{array}$ & 5 & 11 & 24 & 36 & 18 & 25 & 4 & 18 & 0 & 16 & NR & \\
\hline $\begin{array}{l}\text { Jayakumar, } \\
2021^{20}\end{array}$ & $28.2 \pm 5.7$ & $25.8 \pm 2.6$ & & $\mathrm{R}$ & 13 & 9 & 13 & 19 & 4 & 4 & 3 & 2 & NR & \\
\hline
\end{tabular}




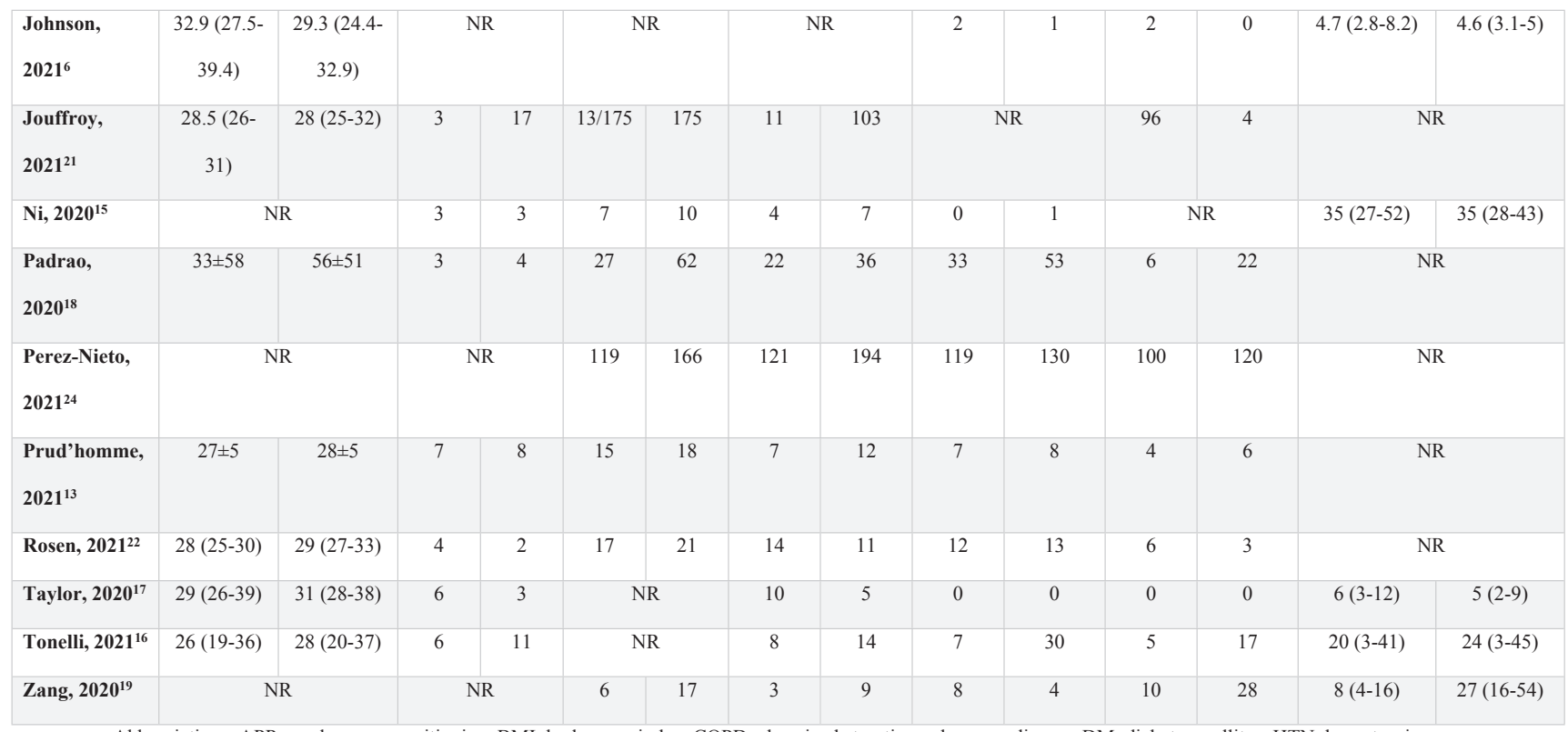

Abbreviations: APP: awake prone positioning, BMI: body mass index, COPD: chronic obstructive pulmonary disease, DM: diabetes mellitus, HTN: hypertension, IQR: interquartile range, n: sample size, NR: not reported, SD: standard deviation. 
